\title{
Control of a servo-hydraulic system utilizing an extended wavelet functional link neural network based on sine cosine algorithms
}

\author{
Shaymaa Mahmood Mahdi, Omar Farouq Lutfy \\ Department of Control and Systems Engineering, University of Technology-Iraq, Baghdad, Iraq
}

\begin{abstract}
Article Info
\section{Article history:}

Received Sep 25, 2021

Revised Nov 30, 2021

Accepted Dec 9, 2021

\section{Keywords:}

Functional link neural network PID controller

Servo-hydraulic system

Sine cosine algorithm

Wavelet neural network

ABSTRACT

Servo-hydraulic systems have been extensively employed in various industrial applications. However, these systems are characterized by their highly complex and nonlinear dynamics, which complicates the control design stage of such systems. In this paper, an extended wavelet functional link neural network (EWFLNN) is proposed to control the displacement response of the servo-hydraulic system. To optimize the controller's parameters, a recently developed optimization technique, which is called the modified sine cosine algorithm (M-SCA), is exploited as the training method. The proposed controller has achieved remarkable results in terms of tracking two different displacement signals and handling external disturbances. From a comparative study, the proposed EWFLNN controller has attained the best control precision compared with those of other controllers, namely, a proportional-integralderivative (PID) controller, an artificial neural network (ANN) controller, a wavelet neural network (WNN) controller, and the original wavelet functional link neural network (WFLNN) controller. Moreover, compared to the genetic algorithm (GA) and the original sine cosine algorithm (SCA), the M-SCA has shown better optimization results in finding the optimal values of the controller's parameters.
\end{abstract}

This is an open access article under the CC BY-SA license.

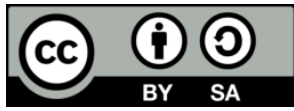

\section{Corresponding Author:}

Omar Farouq Lutfy

Department of Control and Systems Engineering, University of Technology

Baghdad, Iraq

Email: omar.f.lutfy@uotechnology.edu.iq

\section{INTRODUCTION}

Servo-hydraulic systems are essential operating units in many industrial applications due to their high precision, low operating temperatures, low noise, and good repeatability. Moreover, servo-hydraulic systems can attain energy savings of up to $70 \%$ compared to other conventional hydraulic systems [1]. However, these systems are characterized by their highly complex and nonlinear dynamics, and hence, they require precise and powerful controllers to cope with the complexity and nonlinearity of such systems.

The proportional-integral-derivative (PID) controller is one of the most widely used controllers in the industry due to its simple structure and satisfactory performance. Therefore, this controller was broadly employed to control the servo-hydraulic systems. For instance, Wart et al. [2] utilized the PID controller for the position control of an electro-hydraulic system. As the tuning method, the authors used the ZieglerNichols approach to optimize the gains of the PID controller. In another work, Lin et al. [1] proposed a method to control the velocity-pressure switchover point in a servo-hydraulic system using the PID controller. As an intelligent tuning method, Mahdi [3] used the ant colony optimization to find the optimal settings for the PID controller's gains to control a servo-hydraulic system whose model was linearized to 
simplify the control design procedure. Following the same control design, many researchers used different tuning methods to find the optimal gains of the PID controller [4]-[11]. However, in the above-mentioned works, it is worth noticing that controlling the highly complex and nonlinear hydraulic systems by the linear PID controller might not give the best control results, especially when the complex and nonlinear system's model is linearized around a specific operating condition, which means that the controller can perform well only for certain operating conditions and not for other conditions.

To handle this limitation, many researchers employed various computational intelligence techniques to directly control the complex and nonlinear models of the servo-hydraulic systems without the need to make certain simplifications or linearization for the systems models. Among these intelligent techniques, artificial neural networks (ANNs) have been successfully applied to control hydraulic systems. For example, Gao et al. [12] exploited the radial basis function neural network (RBFNN) to control a servo-hydraulic system. The gradient descent (GD) method was used to update the parameters of the RBFNN. Utilizing the model reference adaptive control structure, Yao et al. [13] proposed a control strategy that employs an ANN for the tracking control problem of a servo-hydraulic system. The ANN weights were optimized using a GD procedure. In another work, Diontar et al. [14] proposed to use the nonlinear auto-regression moving average (NARMA) network, which is a type of ANNs, for the position tracking of a hydraulic system using the GD method to optimize the controller's parameters. However, GD techniques have certain limitations including the slow convergence speed, the inclination to getting stuck in local minimum points, and the difficulty of choosing a suitable learning rate [15]. These limitations can be avoided by adopting evolutionary algorithms (EAs) for the optimization task. In particular, EAs can offer remarkable optimization results, as they can escape local minima and find a global solution.

Among several ANN structures, wavelet neural networks (WNNs) that utilize wavelet transform [16]-[18] and functional link neural networks (FLNNs) have distinctive approximation abilities that qualify them to be effective tools for solving different modeling and control problems. To attain better performance, the features of both the WNN and the FLNN can be combined to realize a more powerful structure with better approximation ability [19]-[21].

This paper presents an extended wavelet functional link neural network (EWFLNN) controller to control the servo-hydraulic system. A recently developed EA method, which is called the modified sine cosine algorithm (M-SCA) was applied to optimize the parameters of the EWFLNN controller, which has shown its superiority over other related controllers in controlling the servo-hydraulic systems. The rest of the article is organized according to the following sections: section 2 describes the mathematical modeling of the servo-hydraulic system. The structure of the proposed EWFLNN controller is highlighted in section 3 . Section 4 sheds some light on the M-SCA. The results of the control performance test along with those of two comparative studies are presented and discussed in section 5. Finally, section 6 gives the main conclusions of the present work.

\section{MATHEMATICAL MODELLING OF THE SERVO-HYDRAULIC SYSTEM}

A servo-hydraulic system has several components that are related to each other, as shown in Figure 1. The main working principle is to use a pressurized liquid to control the displacement, velocity, and acceleration of the system's cylinder that starts from tacking the oil from the tank and pressurizes it to control its flow rate through the servo valve reaching the cylinder chambers, where the oil's pressure is transformed into a mechanical force to implement the piston motion for a specific distance. This mechanism is explained in the following sections.

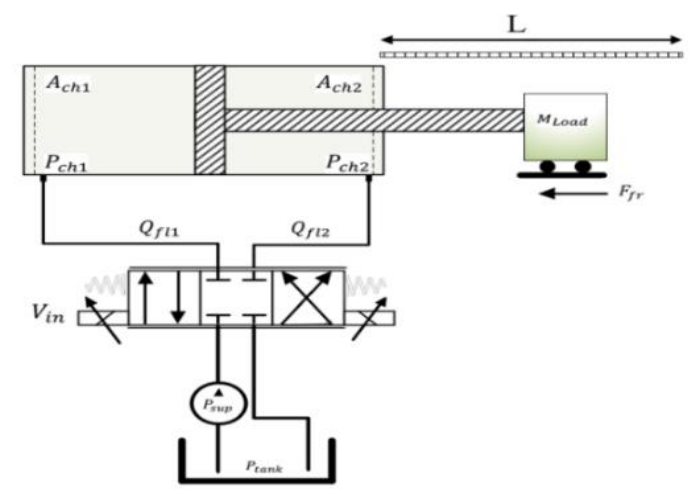

Figure 1. The servo-hydraulic system schematic diagram 


\subsection{Dynamic equations of the hydraulic cylinder}

The mathematical model of the hydraulic cylinder depends on Newton's second law of moving a mass load and taking into consideration the friction forces, as in the [4],

$$
\ddot{y_{a}}=\frac{1}{M_{\text {Load }}}\left(P_{c h 1} A_{c h 1}-P_{c h 2} A_{c h 2}-F_{f r}\right),
$$

where $\left(y_{a}\right)$ is an unknown parameter representing the position of the cylinder piston's load, $\left(M_{\text {Load }}\right)$ represents the mass of the piston's load, and $\left(P_{c h 1}\right)$ and $\left(P_{c h 2}\right)$ denote the hydraulic cylinder's pressures of Chamber 1 and Chamber 2, respectively. In this work, $\left(F_{f r}\right)$, which represents the friction in the hydraulic cylinder, is accounted for as an external force.

The LuGre model is used to represent the equations of the friction, as given in the [4],

$$
\begin{aligned}
& \frac{\mathrm{dz}}{\mathrm{dt}}=\dot{y}_{a}-\frac{\left|\dot{y}_{a}\right|}{\mathrm{g}\left(\dot{y_{a}}\right)} \mathrm{z} \\
& g\left(\dot{y}_{a}\right)=\frac{1}{\sigma_{s t}}\left(\mathrm{~F}_{c o l}+\left(\mathrm{F}_{s t}-\mathrm{F}_{c o l}\right) e^{\left.-\left(\frac{y_{a}}{V s t r}\right)^{2}\right)}\right. \\
& F_{f r}=\sigma_{s t} Z+\sigma_{d a m p} \frac{d z}{d t}+V_{f} \dot{y}_{a}
\end{aligned}
$$

where $\left(\dot{y_{a}}\right)$ signifies cylinder piston's velocity, $F_{f r}$ represents the friction force that is defined by a linear combination of $(z),\left(\frac{\mathrm{dz}}{\mathrm{dt}}\right)$, and the viscous friction. In (4) is the friction's dynamics. The variable $(z)$ represents an internal state, $g\left(\dot{y}_{a}\right)$ defines part of the "steady-state" characteristics for motions of constant velocity, $V s t r$ denotes the Stribeck velocity, $F_{s t}$ denotes the static friction, $F_{c o l}$ is the Coloumb friction, and $V_{f}$ is the viscous friction. As a result, the final friction model is defined by four static parameters, two dynamic parameters, the coefficient of stiffness $\left(\sigma_{s t}\right)$, and coefficient of damping $\left(\sigma_{d a m p}\right)$.

\subsection{Calculations of pressure for the chambers within the cylinder}

The equation of pressures in the cylinder chambers are calculated based on the equations of flow continuity for the servo-valve in the volume between the orifices and their outlets, as given [4],

$$
\begin{aligned}
& \dot{P}_{c h 1}=\frac{\beta e_{1}}{v_{c h 1}}\left(-Q_{f l 1}+A_{c h 1} \dot{y}_{a}-Q_{I L}-Q_{E L 1}\right) \\
& \dot{P}_{c h 2}=\frac{\beta e_{2}}{v_{c h 2}}\left(-Q_{f l 2}+A_{c h 2} \dot{y}_{a}-Q_{I L}-Q_{E L 2}\right)
\end{aligned}
$$

where $\left(Q_{I L}\right)$ is the internal leakage flow, $\left(Q_{E L 1}\right)$ and $\left(Q_{E L 2}\right)$ denote external leakage flows, $\left(A_{c h 1}\right)$ and $\left(A_{c h 1}\right)$ signify areas of the cylinder's piston of Chamber 1 and Chamber 2 , respectively, $\left(v_{\operatorname{ch} 1}\right)$ and $\left(v_{\operatorname{ch} 2}\right)$ represent volumes between each side of the cylinder's chambers, and $\left(\beta e_{1}\right)$ and $\left(\beta e_{2}\right)$ represent bulk modulus for the hydraulic fluids in each side of the piston, respectively.

Volumes' calculations for each cylinder chamber are represented in the equations,

$$
\begin{aligned}
& v_{c h 1}=A_{c h 1} y_{a}+V_{o 1} \\
& v_{c h 2}=A_{c h 2}\left(L-y_{a}\right)+V_{o 2}
\end{aligned}
$$

where $\left(V_{o 1}\right)$ and $\left(V_{o 2}\right)$ denote volumes of the pipeline at Ports 1 and 2, respectively, and $L$ denotes the length of the stroke.

\subsection{Flow-pressure equations of the servo valve}

The servo valve flow rate equations are considered nonlinear equations described by the relationship between the servo valve spool displacement $\left(y_{s}\right)$ and the pressure drop, as given,

$$
\begin{aligned}
& Q_{f l 1}=\left\{\begin{array}{l}
C_{s} y_{s} \sqrt{P_{s u p}-P_{c h 1}}, V_{\text {in }} \geq 0 \\
C_{s} y_{s} \sqrt{P_{c h 1}-P_{\text {tank }}}, V_{\text {in }}<0
\end{array}\right. \\
& Q_{f l 2}=\left\{\begin{array}{l}
C_{s} y_{s} \sqrt{P_{\text {ch } 2}-P_{\text {tank }}}, V_{\text {in }} \geq 0 \\
C_{s} y_{s} \sqrt{P_{\text {sup }}-P_{\text {ch } 2}}, V_{\text {in }}<0
\end{array}\right.
\end{aligned}
$$


where $\left(P_{\text {sup }}\right)$ and $\left(P_{\text {tank }}\right)$ represent the supply and the tank pressure, respectively, and $\left(C_{s}\right)$ represents a parameter that includes the discharge coefficient and the fluid density.

Considering low frequencies of up to $50 \mathrm{~Hz}$, a model of first-order representation can adequately describe the spool dynamics. More precisely, the connection between the position of the spool $\left(y_{s}\right)$ and the input voltage $\left(V_{i n}\right)$ is described by the $(11)$,

$$
G_{s}(s)=\frac{y_{s}}{V_{i n}}=\frac{x_{s d v}}{s+x_{t}}
$$

where $\left(x_{s d v}\right)$ denotes the gain's value, and $\left(x_{t}\right)$ denotes the time constant.

\section{THE EXTENDED WAVELET FUNCTIONAL LINK NEURAL NETWORK (EWFLNN) CONTROLLER}

In this work, the servo-hydraulic system described above is controlled using the control structure shown in Figure 2, in which the EWFLNN acts as a PID-like feedback controller whose parameters can be adjusted by the M-SCA. In particular, the EWFLNN receives three input signals, namely; the control error $e(k)$, the rate of change in error $\Delta e(k)$, and the summation of errors $\Sigma e(k)$. As the actuating signal, the EWFLNN controller generates the control input $u(k)$ to manipulate the displacement response of the servohydraulic system. The controller's parameters are optimized by the M-SCA based on minimizing the integral square of errors (ISE) criterion given in (12),

$$
I S E=\frac{1}{2} \sum_{k=1}^{N} e^{2}(k)
$$

where $e(k)=y_{d}(k)-y_{a}(k), N$ is the number of samples, $y_{d}(k)$ is the desired displacement, and $y_{a}(k)$, is the actual system's displacement at time sample $\mathrm{k}$.

To improve the approximation performance of a previously developed structure [21], a modification was made in this work by adding the input variables together with a bias weight to the output node, as illustrated in Figure 3, which depicts the proposed EWFLNN structure. Particularly, the input variables are connected through the adjustable parameters $a_{1}, a_{2}, \ldots, a_{N i}$ while the bias weight is connected through the adjustable parameter $b$, as shown in Figure 3. This modification has significantly enhanced the approximation accuracy of the resulting network compared to the original structure in controlling the servo-hydraulic system, as will be seen in the comparative study of section 5.2.

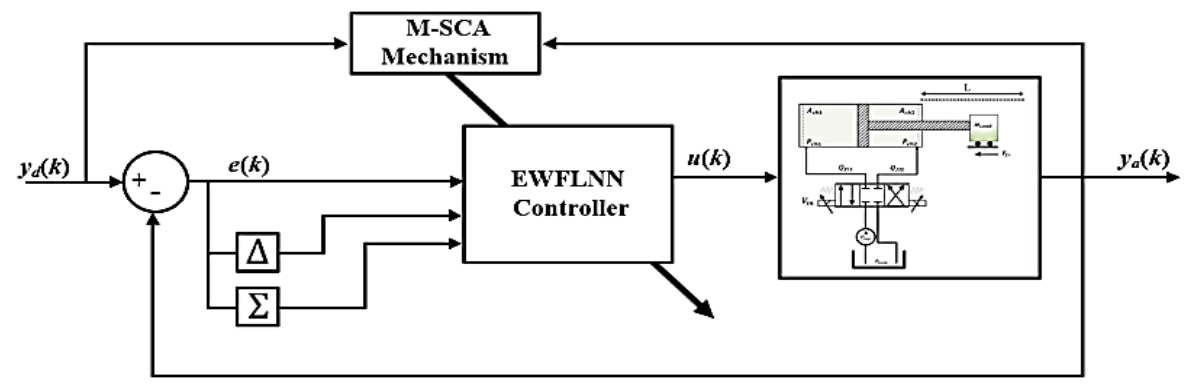

Figure 2. A block diagram of the control structure to control the servo-hydraulic system

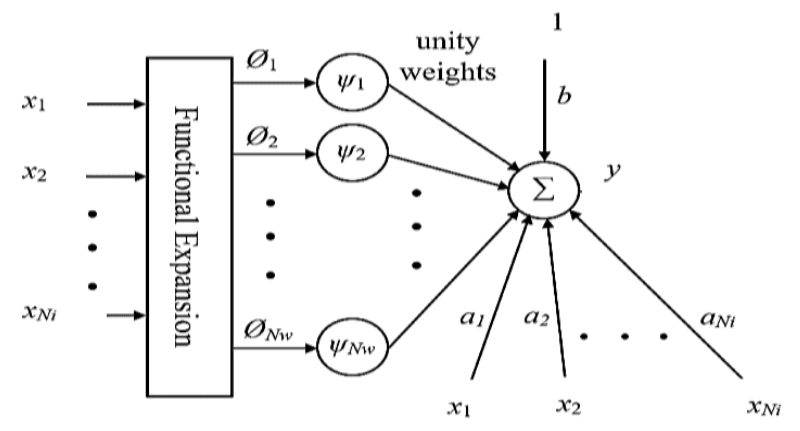

Figure 3. Structure of the EWFLNN controller 
Referring to Figure 3, the proposed EWFLNN structure is composed of three layers including a functional expansion layer, a wavelet layer, and an output layer. Particularly, the functional expansion layer is responsible for increasing the dimensions of the input space utilizing trigonometric function terms, as described by (13),

$$
\phi=\left[\phi_{1}, \phi_{2}, \ldots, \phi_{N w}\right]=\left[x_{1}, \cos \left(\pi x_{1}\right), \sin \left(\pi x_{1}\right), \ldots, x_{N i}, \cos \left(\pi x_{N i}\right), \sin \left(\pi x_{N i 1}\right)\right],
$$

where $N_{w}$ and $N_{i}$ denote the number of functional expansion terms and the number of input variables, respectively. Subsequently, each output from this layer enters a wavelet node in the wavelet layer, which performs the following operator,

$$
z_{j}=d_{j} \phi_{j}-t_{j}
$$

where $j=1,2, \ldots, N_{w}, d_{j}$ and $t_{j}$ signify the dilation and the translation factors of the $j$ th node in the wavelet layer, respectively, and $\phi_{j}$ denotes the $j$ th output resulting from the functional expansion layer. After that, the RASP1 wavelet function was employed to compute the output of each node in the wavelet layer according to the following expression,

$$
\psi_{j}\left(z_{j}\right)=\frac{z_{j}}{\left(z_{j}^{2}+1\right)^{2}}
$$

where $z_{j}$ represents the result of (14). Next, the output of each wavelet node is connected to the output node via a unity weight. Finally, the output node produces the network's output as given (16).

$$
y=\sum_{j=1}^{N w} \psi_{j}+\sum_{i=1}^{N i} a_{i} x_{i}+b,
$$

\section{THE MODIFIED SINE COSINE ALGORITHM}

The sine cosine algorithm (SCA) is a stochastic population-based technique that was developed by Mirjalili in 2016 [22]. As an effective evolutionary search algorithm, the SCA has been successfully applied for handling various optimization problems [23]-[25]. In this work, a modified version of the original SCA, which was developed in [21], was exploited to train the EWFLNN controller. This algorithm was called the M-SCA and it has shown superior optimization results compared with other algorithms, including the original SCA. More specifically, the M-SCA was applied for optimizing the parameters of the EWFLNN controller according to the following procedure:

- Step 1: Initialize the maximum number of iterations and the number of candidate solutions in the M-SCA.

- Step 2: Generate randomly the candidate solutions representing the modifiable parameters of the controller.

- Step 3: In this step, the cost function defined in (12) is calculated for each solution.

- Step 4: Find the best solution compared to other solutions. This solution is assigned as the destination point.

- Step 5: In this step, the values of four random parameters $r_{1}, r_{2}, r_{3}$, and $r_{4}$ are updated.

- Step 6: The position of each candidate solution is updated according to the following equation.

$$
X_{i}^{t+1}=\left\{\begin{array}{l}
X_{i}^{t}+r_{1} \times \sin \left(r_{2}\right) \times\left|r_{3} P_{i}^{t}-X_{i}^{t}\right|, r_{4}<0.5 \\
X_{i}^{t}+r_{1} \times \cos \left(r_{2}\right) \times\left|r_{3} P_{i}^{t}-X_{i}^{t}\right|, r_{4} \geq 0.5
\end{array}\right.
$$

Where $X_{i}^{t}$ denotes a solution position in the $i$ th dimension at the $t$ th iteration, $r_{2}, r_{3}$, and $r_{4}$ represent random variables, $P_{i}$ is the position of the destination point in the $i$ th dimension, and | | is the absolute value. The variable $r_{1}$ decides the next movement's direction of each solution according to the position of the destination point $P$. To achieve an appropriate balance between the exploration and the exploitation abilities of the algorithm, $r_{1}$ is computed adaptively using (18),

$$
r_{1}=a-t \frac{a}{T}
$$

where $a$ is a constant, $t$ is the current iteration, and $T$ is the maximum number of iterations. On the other hand, the values of $r_{2}, r_{3}$, and $r_{4}$ are generated randomly from the intervals $[0,2 \pi],[0,2]$, and [0, 1], respectively [22].

- Step 7: In this step, the solutions are ranked according to their cost function starting from the solution with the best cost function to the solution with the worst cost function.

- Step 8: Substitute the worst $n$ solutions by $n$ new solutions, where $n$ was set to 20 in this work, and the solutions were produced according to the (19), 


$$
X_{i, j}^{t+1}=P_{j}^{t}+\mu_{i, j}\left(X_{m 1, j}^{t}-X_{m 2, j}^{t}\right)
$$

where $i$ indicates the position and $j$ indicates the dimension of solution $X, P$ is the best solution, $m_{1}$ and $m_{2}$ are two integer numbers randomly chosen between 1 and the maximum number of solutions and they should also be different from the current solution's position, $i$, and $\mu_{i, j}$ is a random number generated from $[-1,1]$.

- Step 9: A solution is randomly generated in this step and its cost function is calculated. If the cost function is worse than that of the worst solution, the worst solution is substituted by the position of the destination point. Otherwise, the newly generated solution replaces the worst solution.

- Step 10: If the maximum number of iterations is reached, the algorithm is stopped and the best solution achieved so far is utilized as the optimized parameters of the EWFLNN controller. Otherwise, the above procedure is repeated starting from Step 3.

\section{SIMULATION RESULTS}

This section aims at assessing the control accuracy of the proposed EWFLNN controller to control the servo-hydraulic system described in section 2. As the training algorithm, the M-SCA was applied using 60 solutions and 30 iterations for all the controllers considered in this section. These settings were sufficient to attain the required control performance.

\subsection{Control performance tests}

To evaluate the performance of the proposed EWFLNN controller, several simulation tests were conducted using the mathematical model of the servo-hydraulic system described in section 2 . For this purpose, an M-file in the Matlab software was utilized to optimize the controller parameters, while the nonlinear servo-hydraulic system's model with the servo valve was implemented using the Simulink environment, as illustrated in Figure 4. Figure 5 depicts the open-loop response of the servo-hydraulic system's model described in section 2 using the parameters' values listed in Table 1 [4].

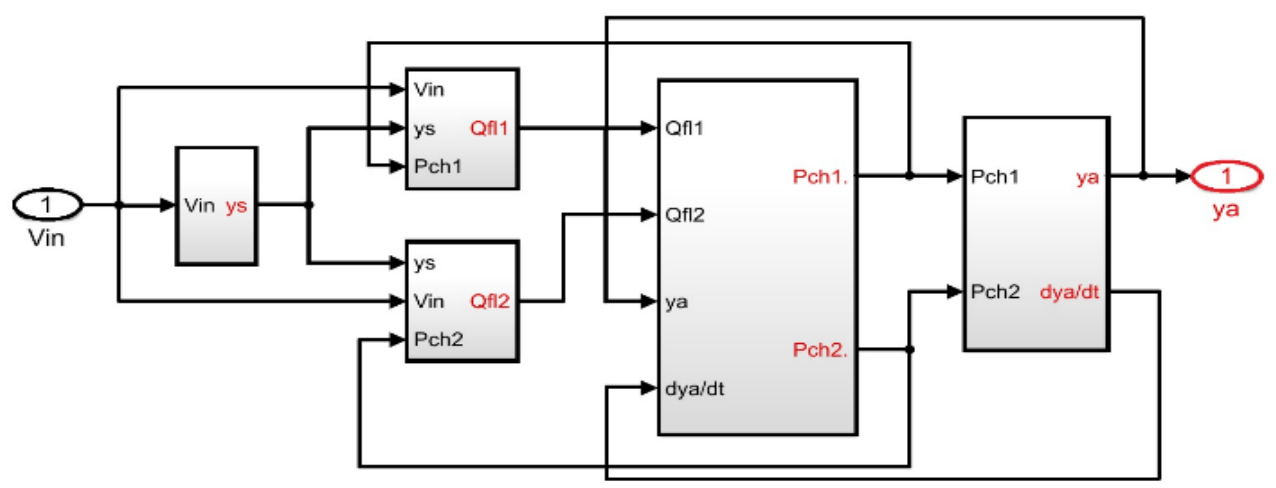

Figure 4. A block diagram of the servo-hydraulic system in Simulink

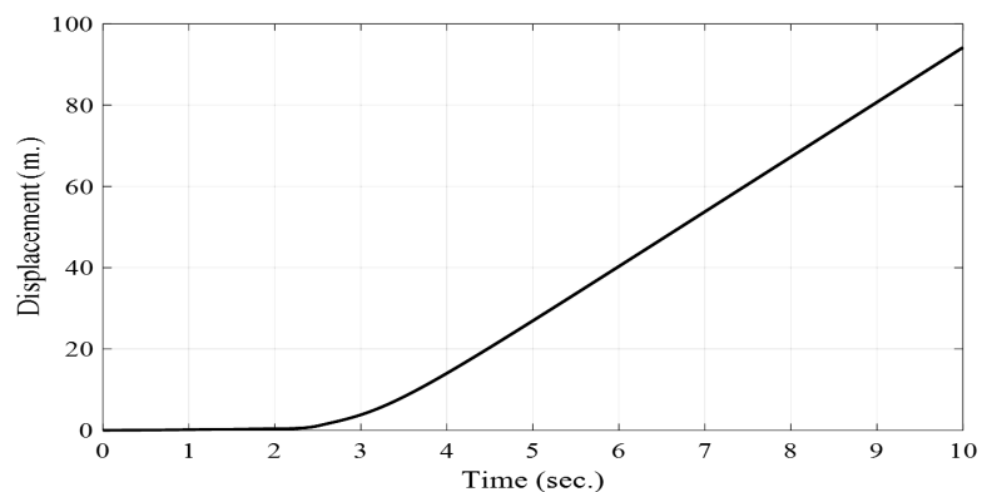

Figure 5. The open-loop displacement response of the servo-hydraulic system 
Table 1. Parameters' values of the servo-hydraulic system [4]

\begin{tabular}{lc}
\hline \multicolumn{1}{c}{ Parameter } & value \\
\hline Area of Chamber 1 $\left(A_{\text {ch } 1}\right)$ & $8.04 \times 10^{-4} \mathrm{~m}^{2}$ \\
Area of Chamber 2 $\left(A_{\text {ch } 2}\right)$ & $4.24 \times 10^{-4} \mathrm{~m}^{2}$ \\
Mass load $\left(M_{\text {Load }}\right)$ & $210 \mathrm{~kg}$ \\
Piston's length $(\mathrm{L})$ & $1 \mathrm{~m}$ \\
Pressure of the supply $\left(P_{\text {sup }}\right)$ & $14 \mathrm{MPa}$ \\
Pressure of the tank $\left(P_{\text {tank }}\right)$ & $0.9 \mathrm{MPa}$ \\
Columbic friction $\left(F_{\text {col }}\right)$ & $247.804 \mathrm{~N}$ \\
Static friction $\left(F_{\text {st }}\right)$ & $7485.084 \mathrm{~N}$ \\
Viscous friction $\left(V_{f}\right)$ & $376.613 \mathrm{Ns} / \mathrm{m}$ \\
Stribeck velocity $\left(V_{s t r}\right)$ & $0.026318 \mathrm{~m} / \mathrm{s}$ \\
\hline
\end{tabular}

Figure 5 clearly indicates that the system has an unstable open-loop response. Therefore, the proposed EWFLNN controller was applied to control this system. In particular, two control performance tests were conducted to assess the control result of the EWFLNN controller to make the output of the servohydraulic system follow two different reference signals. Figure 6 demonstrates the system's response in tracking the first reference signal, which is a changing step signal. As it is evident from Figure 6, the EWFLNN controller has achieved remarkable control accuracy with zero steady-state error. Figure 7 shows the output response of the servo-hydraulic system controlled by the EWFLNN controller to track another reference signal. Figure 7 clearly demonstrates that the controller has done well in following the desired signal with zero-steady state error.

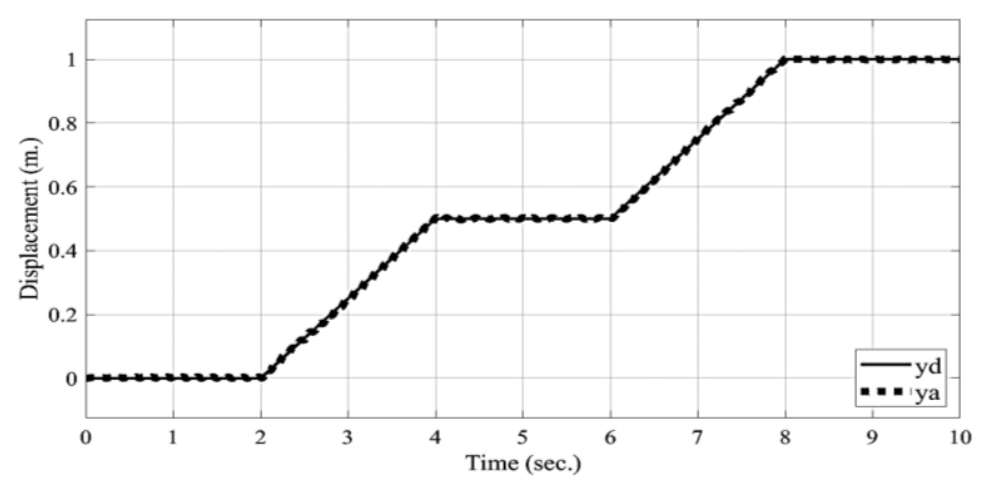

Figure 6. The servo-hydraulic system response for the first reference input

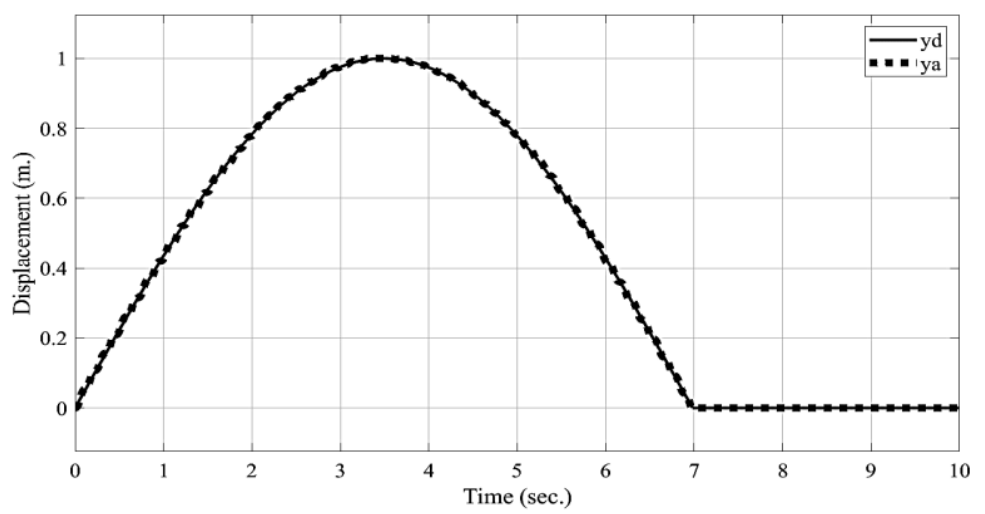

Figure 7. The servo-hydraulic system response for the second reference input

In order to assess the controller's robustness ability, a disturbance test was performed by injecting an external disturbance of $(+0.4)$ for the period from 4 to 5 seconds and $(-0.4)$ for the period from 5 to 6 seconds. Figure 8(a) depicts the result of this test. Moreover, Figure 8(b) shows the output response for the 
same disturbance of Figure 8(a) plus a uniform random disturbance that continues for the entire simulation time. From both Figurs 8(a) and (b) it can be seen that the controller was able to suppress the effect of the disturbance and it brought the system's response back to the desired reference signal.

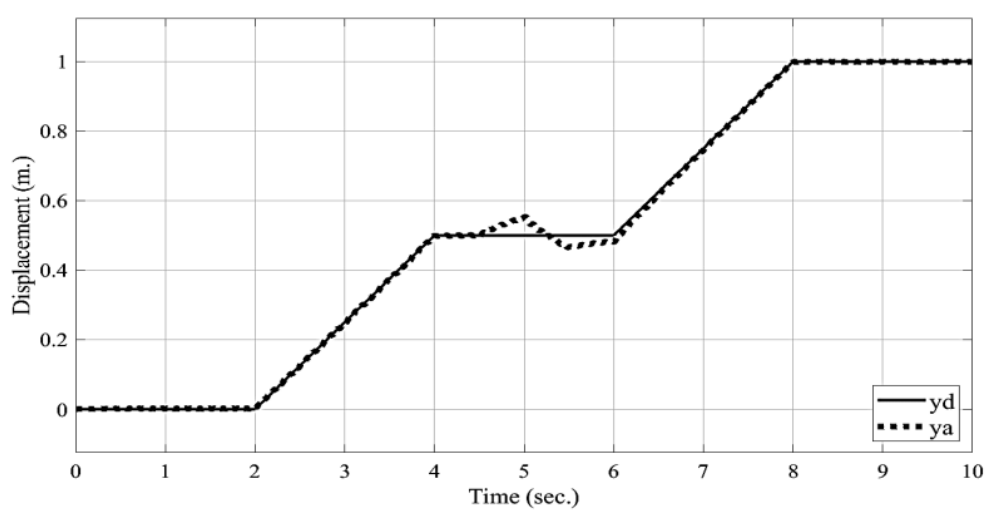

(a)

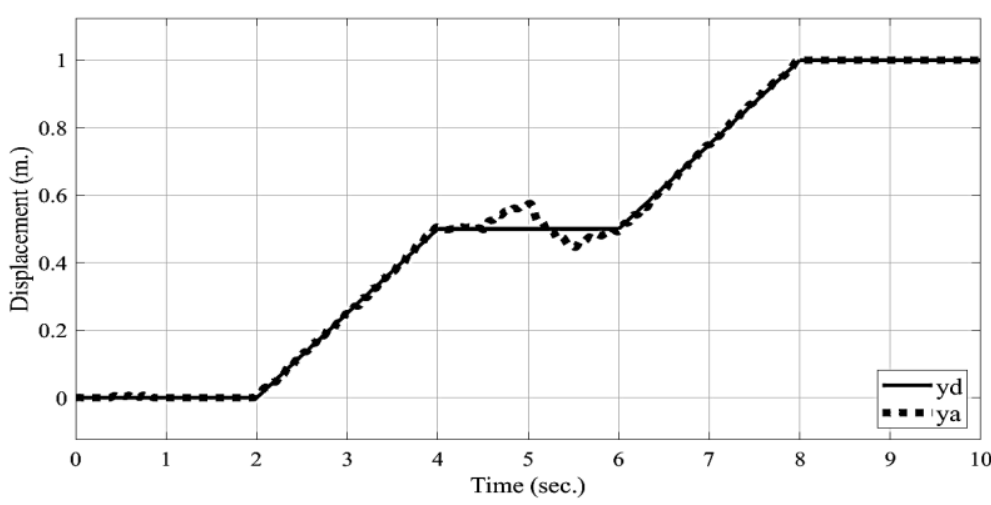

(b)

Figure 8. The servo-hydraulic system response in handling: (a) the $\mp 0.4$ external disturbance and (b) both the $\mp 0.4$ external disturbance and the $\mp 0.1$ uniform random disturbance

\subsection{A comparsion study with other types of controllers}

In this section, the control performance of the EWFLNN controller was compared with those of other controllers including, a proportional-integral-derivative (PID) controller, an artificial neural network (ANN) controller, a wavelet neural network (WNN) controller, and the original wavelet functional link neural network (WFLNN) controller. All the above controllers were trained by the M-SCA with the same settings mentioned in section 5. In order to take the stochastic nature of the M-SCA into consideration, 10 runs were carried out for each controller and the average result was adopted. Table 2 displays the outcome of the comparative study. From Table 2, it is obvious that the EWFLNN controller has resulted in the best control accuracy in terms of achieving the least value for the ISE cost function. In this regard, it is worth noticing that the proposed modification made in the EWFLNN has significantly improved the performance of the original WFLNN controller.

Table 2. Comparison results of the PID, the ANN, the WNN, the original WFLNN, and the proposed EWFLNN controller

\begin{tabular}{lc}
\hline \multicolumn{1}{c}{ Controller type } & ISE Criterion (average of 10 runs) \\
\hline PID controller & $7.27 \times 10^{-5}$ \\
ANN controller & $64.22 \times 10^{-5}$ \\
WNN controller & $33.13 \times 10^{-5}$ \\
WFLNN controller & $121.21 \times 10^{-5}$ \\
EWFLNN controller & $5.6 \times 10^{-5}$ \\
\hline
\end{tabular}




\subsection{A comparsion study with other optimization techniques}

In this section, the optimization result of the M-SCA has been compared with those of the genetic algorithm (GA), which is considered as one of the most powerful and widely used evolutionary algorithms [26]-[28], and the original sine cosine algorithm (SCA). Using the same comparison analysis of the previous section, 10 runs were made for each algorithm and the average of these runs was taken. Table 3 illustrates the result of this test, where it is clear that the M-SCA has resulted in the fewest ISE value compared to those of the GA and the SCA.

Table 3. Comparison results of the GA, the SCA, and the M-SCA acting as the training methods

\begin{tabular}{cc}
\hline Optimization Method & ISE Criterion (average of 10 runs) \\
\hline GA & $10.4 \times 10^{-5}$ \\
SCA & $27.6 \times 10^{-5}$ \\
M-SCA & $5.62 \times 10^{-5}$ \\
\hline
\end{tabular}

\section{CONCLUSION}

In this paper, an extended wavelet functional link neural network structure was put forward to control the highly complex and nonlinear servo-hydraulic system. A recently developed optimization method, namely the M-SCA, was employed to find the optimal settings for the proposed controller, which has attained remarkable control accuracy in tracking two different displacement signals. The results of a comparative study involving other types of controllers revealed the superiority of the EWFLNN controller. In addition, MSCA has achieved better optimization results in finding the optimal values of the controller's parameters compared to the GA and the SCA.

\section{REFERENCES}

[1] C. Y. Lin, F. C. Shen, K. T. Wu, H. H. Lee, and S. J. Hwang, "Injection molding process control of servo-hydraulic system," Applied Sciences MDPI, vol. 10, no. 71, Dec. 2019, doi:10.3390/app10010071.

[2] G. Wart, P. Ranjan, M. Bhola, S. K. Mishra, and J. Das, "Position control and performance analysis of hydraulic system using two pump-controlling strategies," Proc IMechE Part I:J Systems and Control Engineering sagepub, vol. 1, no. 13, 2018, doi: $0.1177 / 0959651818813233$.

[3] Sh. M. Mahdi, K. S. Khalid, and Sh. M. Mahdi, "PID controller tuning using ant colony method for servo hydraulic system," Journal of Mechanical Engineering Research and Developments (JMERD), vol. 42, no. 4, pp. 136-140, July 2019, doi: 10.26480/jmerd.04.2019.148.152.

[4] Sh. M. Mahdi, "Controlling a nonlinear servo hydraulic system using PID controller with a genetic algorithm tool," Iraqi Journal of Computers, Communication, Controls \& Systems Engineering (IJCCCE), vol. 12, no. 1, 2012.

[5] M. El-S. M. Essa, M. AS. Aboelela, and M. A. M. Hassan, "Position control of hydraulic servo system using proportionalintegral-derivative controller tuned by some evolutionary techniques," Journal of Vibration and Control, vol. 1, no. 12, 2014, doi: $10.1177 / 1077546314551445$

[6] S. M. Othman, M. F. Rahmat, and S. Md. Rozaliand, "PID parameters optimization using PSO technique for nonlinear electro hydraulic actuator," Journal Teknologi (Sciences \& Engineering), vol. 77, no. 28, 2015, doi: 10.11113/jt.v77.6793.

[7] T. Samakwong and W. Assawinchaichote, "PID controller design for electro-hydraulic servo valve system with genetic algorithm," in Proc 2016 International Electrical Engineering Congress, iEECON2016, 2-4 March 2016, doi: 10.1016/j.procs.2016.05.023.

[8] M. Alhanjouri, "Modern optimization techniques for PID parameters of electrohydraulic servo control system," International Journal on Recent and Innovation Trends in Computing and Communication, vol. 5, no. 3, pp. 71-79, March 2017.

[9] Y. Fan, J. Shao, and G. Sun, "Optimized PID controller based on beetle antennae search algorithm for electro-hydraulic position servo control system," Sensors, vol. 19, no. 2727, June 2019, doi: 10.3390/s19122727.

[10] C. C. Soon, R. Ghazali, S. H. Chong, C. M. Shern, Y. Md. Sam, and Z. Has, "Efficiency and performance of optimized robust controllers in hydraulic system," International Journal of Advanced Computer Science and Applications, vol. 11, no. 6, 2020, doi: 10.14569/IJACSA.2020.0110650.

[11] Y. Fan, J. Shao, G. Sun, and X. Shao, "Proportional-Integral-Derivative controller design using an advanced lévy-flight salp swarm algorithm for hydraulic systems," Energies, vol.13, no. 459, Januray 2020, doi: 10.3390/en13020459.

[12] Q. Gao, Y. Hou, K. Li, Z. Sun, C. Wang, and R. Hou, "Neural network based active disturbance rejection control of a novel electrohydraulic servo system for simultaneously balancing and positioning by isoactuation configuration," Hindawi Publishing Corporation Shock and Vibration, vol. 9, 2016, doi: 10.1155/2016/4921095.

[13] Z. Yao, J. Yao, F. Yao, Q. Xu, M. Xu, and W. Deng, "Model reference adaptive tracking control for hydraulic servo systems with nonlinear neural-networks," ISA Transactions, vol. 100, pp. 396-404, May 2020, doi: 10.1016/j.isatra.2019.11.027.

[14] N. Diontar, C. A. Otieno, and S. I. Kamau, "Electro-hydraulic position tracking using NARMA neural controller," International Journal of Recent Technology and Engineering (IJRTE), vol. 9, no. 1, May 2020, doi: 10.35940/ijrte.F8544.059120.

[15] S. Chakravarty and P. K. Dash, "A PSO based integrated functional link net and interval type-2 fuzzy logic system for predicting stock market indices," Applied Soft Computing, vol. 12, no. 2, pp. 931-941, 2012, doi: 10.1016/j.asoc.2011.09.013.

[16] N. Khannaa, V. Kumarb, and S. K. Kaushik, "Wavelet packet approximation," Integral Transforms and Special Functions, UK Limited, trading as Taylor \& Francis Group, vol. 27, no. 9, pp. 698-714, June 2016, doi: 10.1080/10652469.2016.1189912.

[17] N. Khanna, S. K. Kaushik, and A. M. Jarrah, "Wavelet packets: Uniform approximation and numerical integration," International Journal of Wavelets, Multiresolution and Information Processing, vol. 18, no. 2, 2020, doi: 10.1142/S0219691320500046.

[18] I. Daubechies, "Ten Lectures on Wavelets, " Society for Industral and Applied Mathematics, 3600 University City Science Center Philadelphia, PA, United States, 1992. 
[19] C. F. Hsu, “A self-evolving functional-linked wavelet neural network for control applications,” Applied Soft Computing, vol. 13, no. 11, pp. 4392-4402, Nov. 2013, doi: 10.1016/j.asoc.2013.06.012.

[20] A. Gotmare, R. Patidar, and N. V. George, "Nonlinear system identification using a cuckoo search optimized adaptive Hammerstein model," Expert Systems with Applications, vol. 42, no. 5, pp. 2538-2546, Apr. 2015, doi: 10.1016/j.eswa.2014.10.040.

[21] O. F. Lutfy, "A wavelet functional link neural network controller trained by a modified sine cosine algorithm using the feedback error learning strategy," Journal of Engineering Science and Technology, vol. 15, no. 1, pp. 709-727, 2020.

[22] S. Mirjalili, "SCA: A sine cosine algorithm for solving optimization problems," Knowledge-Based Systems, vol. 96, pp. 120-133, March 2016, doi: 10.1016/j.knosys.2015.12.022.

[23] G. Kaur and J. S. Dhillon, "Economic power generation scheduling exploiting hill-climbed Sine-Cosine algorithm," Applied Soft Computing, vol. 111, (107690), 2021, doi: 10.1016/j.asoc.2021.107690.

[24] Y. Li, Y. Zhao, and J. Liu, "Dynamic sine cosine algorithm for large-scale global optimization problems," Expert Systems with Applications, vol. 177, Nov. 2020, doi: 10.1016/j.asoc.2021.107690.

[25] F. Lyu, X. Fan, F. Ding, and Z. Chen, "Prediction of the axial compressive strength of circular concrete-filled steel tube columns using sine cosine algorithm-support vector regression," Composite Structures, vol. 273, Oct. 2021, doi: 10.1016/j.compstruct.2021.114282.

[26] S. Pandey, S. H. Saeed, and N. R. Kidwai, "Simulation and optimization of genetic algorithm-artificial neural network based air quality estimator," Indonesian Journal of Electrical Engineering and Computer Science (IJEECS), vol. 19, no. 2, Aug. 2020, doi: 10.11591/ijeecs.v19.i2.pp775-783.

[27] H. Suhaimi, S. I. Suliman, A. F. Harun, R. Mohamad, Y. W. M. Yusof, and M. Kassim, "Genetic algorithm for intrusion detection system in computer network," Indonesian Journal of Electrical Engineering and Computer Science (IJEECS), vol. 19, no. 3, Sep. 2020, doi: 10.11591/ijeecs.v19.i3.pp1670-1676.

[28] Z. A. Ali, S. A. Rasheed, and N. N. Ali, "An enhanced hybrid genetic algorithm for solving traveling salesman problem," Indonesian Journal of Electrical Engineering and Computer Science (IJEECS), vol. 18, no. 2, May 2020, doi: 10.11591/ijeecs.v18.i2.pp1035-1039.

\section{BIOGRAPHIES OF AUTHORS}
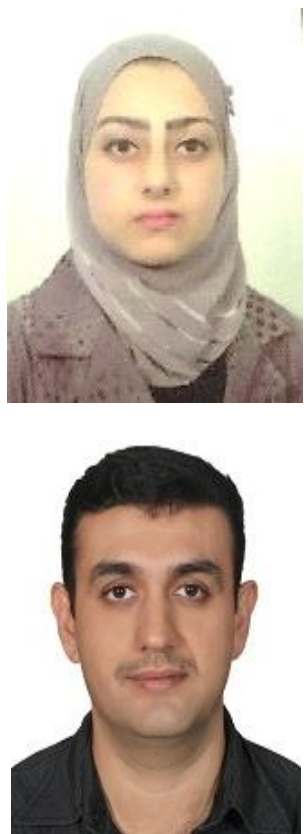

Shaymaa Mahmood Mahdi (D) II SC P received her B.Sc. degree in Control and Systems Engineering and the M.Sc. degree in Mechatronics Engineering from the University of Technology-Iraq, in 2003 and 2008, respectively. She is currently a full-time Assistant Professor, Head of the Quality Assurance \& University Performance Unit, Department of Control and Systems Engineering, University of Technology-Iraq. Her current research interests include hydraulic and pneumatic systems, robotics and intelligent controllers. She can be contacted at email: shaymaa.m.mahdi@uotechnology.edu.iq.

Omar Farouq Lutfy (D) SI SC P received the Ph.D. degree in Control and Automation Engineering at the Department of Electrical and Electronic Engineering, University Putra Malaysia (UPM), Malaysia in 2011. He received the B.Sc. degree in Computer Engineering and the M.Sc. degree in Mechatronics Engineering at the Control and Systems Engineering Department, University of Technology-Iraq in 2000 and 2003, respectively. He is currently working as a professor at the Control and Systems Engineering Department, University of Technology-Iraq. $\mathrm{He}$ is the author and co-author of more than thirty papers published in international journals and conferences. His research interests include intelligent control, artificial intelligence algorithms, and system identification techniques. He can be contacted at email: omar.f.lutfy@uotechnology.edu.iq. 